\title{
The Signal Peptide of the Medicago truncatula Modular Nodulin MtNOD25 Operates as an Address Label for the Specific Targeting of Proteins to Nitrogen-Fixing Symbiosomes
}

\author{
Natalija Hohnjec, ${ }^{1,2}$ Frauke Lenz, ${ }^{1}$ Vera Fehlberg, ${ }^{1}$ Martin F. Vieweg, ${ }^{1}$ Markus C. Baier, ${ }^{1}$ \\ Bettina Hause, ${ }^{3}$ and Helge Küster ${ }^{1,2}$ \\ ${ }^{1}$ Institute for Genome Research and Systems Biology (IGS) and ${ }^{2}$ International Graduate School in Bioinformatics and \\ Genome Research, Center for Biotechnology (CeBiTec), Bielefeld University, D-33594 Bielefeld, Germany; ${ }^{3}$ Leibniz Institute \\ of Plant Biochemistry, P.O. Box 110432, D-06018 Halle/Saale, Germany
}

Submitted 31 July 2008. Accepted 17 September 2008.

The nodule-specific MtNOD25 gene of the model legume Medicago truncatula encodes a modular nodulin composed of different repetitive modules flanked by distinct $\mathrm{N}$ - and C-termini. Although similarities are low with respect to all repetitive modules, both the $\mathrm{N}$-terminal signal peptide (SP) and the $C$-terminus are highly conserved in modular nodulins from different legumes. On the cellular level, MtNOD25 is only transcribed in the infected cells of root nodules, and this activation is mediated by a 299-bp minimal promoter containing an organ-specific element. By expressing mGFP6 translational fusions in transgenic nodules, we show that MtNOD25 proteins are exclusively translocated to the symbiosomes of infected cells. This specific targeting only requires an $\mathrm{N}$-terminal MtNOD25 SP that is highly conserved across a family of legume-specific symbiosome proteins. Our finding sheds light on one possible mechanism for the delivery of host proteins to the symbiosomes of infected root nodule cells and, in addition, defines a short molecular address label of only 24 amino acids whose $\mathrm{N}$-terminal presence is sufficient to translocate proteins across the peribacteroid membrane.

Additional keywords: alternative splicing, confocal laser-scanning microscopy, mRFP.

The root nodule symbiosis between legumes and rhizobial prokaryotes represents the most intensively studied nitrogenfixing plant-microbe interaction (Crespi and Galvez 2000; Schultze and Kondorosi 1998). This extremely host-specific symbiosis is initiated in the root cortex (Geurts and Bisseling 2002) after the perception of rhizobial Nod factors through LysM-domain-containing receptor kinases (Cullimore and Dénarié 2003; Oldroyd and Downie 2006). Subsequent signal transduction triggers the expression of plant genes located downstream of the Nod-factor perception pathway, ultimately resulting in root nodule organogenesis.

Corresponding author: H. Küster; E-mail: Helge.Kuester@ Genetik.UniBielefeld.de

* The $\boldsymbol{e}$-Xtra logo stands for "electronic extra" and indicates three supplemental figures are published online.
Root nodules are colonized by rhizobia through tubular infection threads (Brewin 1991). Subsequent to an endocytotic uptake of rhizobia at the infection thread tips, cells from the inner nodule zones house nitrogen-fixing microsymbionts in transient organelle-like structures termed symbiosomes (Parniske 2000). Here, rhizobia differentiate into bacteroids capable of carrying out the conversion of dinitrogen to ammonia. To drive bacteroid energy metabolism, legumes supply dicarboxylic acids to the endosymbionts via specific dicarboxylic acid transporters located in a specialized symbiotic interface, the largely plant-derived peribacteroid membrane (PBM) (Day et al. 2001). In exchange, ammonia is released through PBM channels (Tyerman et al. 1995) to the plant cytoplasm, where it is incorporated into glutamine by glutamine synthetase (Carvalho et al. 2003). In addition to the nitrogen-fixing bacteroid, the PBM surrounds the acidic peribacteroid space (PBS) (Day et al. 2001; Udvardi and Day 1997). Ultimately, it is the coordinated exchange of carbon for nitrogen metabolites across the PBS and PBM that enables legumes to colonize nitrogendepleted soils, a property exploited in sustainable agriculture (Graham et al. 2004).

An early goal in research on nitrogen-fixing symbioses was the identification of plant genes that initiate and support nodule development. Classical methods used to address this question were differential display and subtractive hybridization techniques. Although these approaches identified dozens of nodulation-related genes in different legumes (Gamas et al. 1996; Pawlowski 1997; Perlick and Pühler 1993), recent genome-enabled studies widened our view of symbiosis-related gene expression by identifying several hundred nodule-activated genes (Colebatch et al. 2004; El Yahyaoui et al. 2004; Fedorova et al. 2002; Journet et al. 2002; Küster et al. 2004). Nodule-specific proteins were traditionally designated nodulins (van Kammen 1984) and were discriminated into early and late nodulins according to their time of synthesis during nodule development. For most nodulin genes, a biological function remains to be assigned.

In a study on nodulation-related genes of broad bean (Vicia faba L.), 19 early and late nodulin genes were identified by differential hybridization (Perlick and Pühler 1993). Among those, the VfNOD28/32 gene encoded modular nodulins composed of different repetitive modules flanked by distinct $\mathrm{N}$ and C-termini (Küster et al. 1994, 1996), reminiscent of the MsNOD25 gene encoding nodulin-25 of Medicago sativa 
(Kiss et al. 1990a). Interestingly, the number and order of repetitive modules was species specific, indicating that the function of these proteins was related to the individual modules (Küster et al. 1999). In Vicia spp., genes specifying modular nodulins were exclusively expressed in the nitrogen-fixing zone, around the onset of nitrogen fixation (Küster et al. 1996, 1999). Expression in nonsymbiotic tissues was not reported for VfNOD28/32 or for homologous genes from $V$. hirsuta (Küster et al. 1999), M. sativa (Kiss et al. 1990a), or Galega orientalis (Kaijalainen et al. 2002).

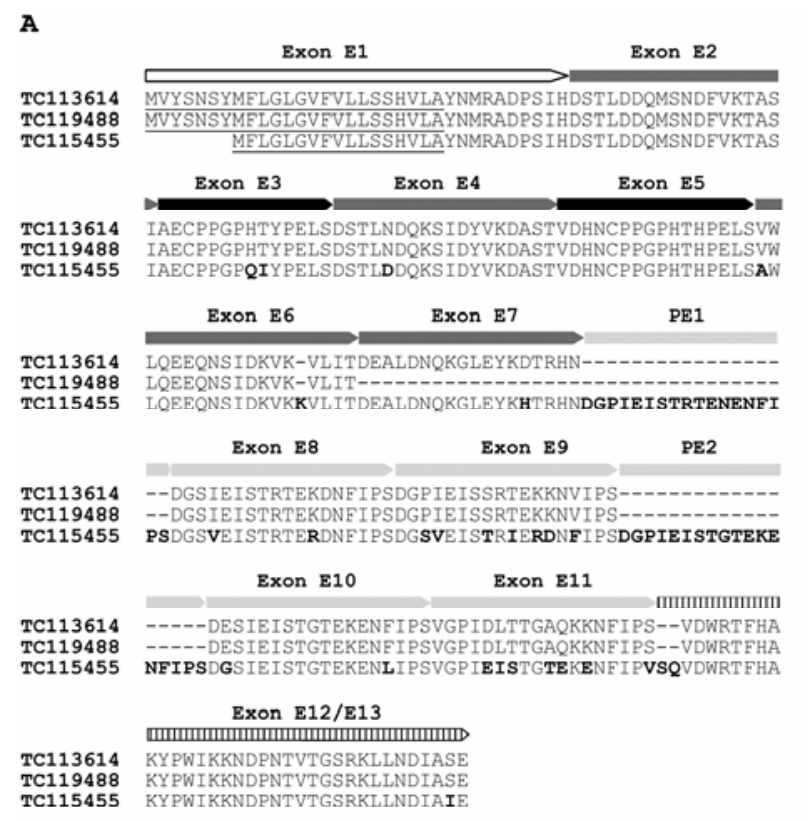

B

$N$ module

Repeat 1 module

MVYSNSYMELGLGVFVLLSSHVLAYNMRADPSIH

Repeat 2 module TVDHNCPPGPHTHPELS DSTLDDQMSNDFVKTAS I DSTLNDQKSIDYVKDAS VWLQEEQNSIDKVKVLI? DEALDNQKGLEYKDTRHN

Repeat 3 module DGSIEISTRTEKDNFIPS DGPIEISSRTEKKNFIPS DESIEISTGTEKENFIPS
VGPIDLTTGAOKKNFIPS

$c$ module

VDWRTFHAKYPWIKKNDPNTVTGSRKLLNDIASE

C

C module (MtNOD25) VDWRTFHAKYPWIKKNDPNTVTGSRKLL-NDIASE

$C$ module (MsNOD25) VDWRTFHAKYPWIKKDGPNTVTGSRKLL-NDIAIK

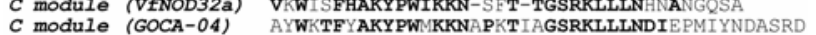

Fig. 1. Sequence properties of modular nodulins from Medicago truncatula cvs. Jemalong and R108. A, Comparison of nodulin-25 proteins encoded by the MtNOD25 tentative consensus sequences (TCs) TC113614, TC119488, and TC115445. Modules identified are indicated by arrows. White arrow, N module; black arrow, repeat 1 module; dark-gray arrow, repeat 2 module; light-gray arrow, repeat 3 module; striped arrow, $\mathrm{C}$ module. Alterations in the deduced amino acid sequence of TC115445 ( $M$. truncatula cv. R108) as opposed to TC113614 and TC119488 (both M. truncatula cv. Jemalong) are marked in bold, and predicted signal peptides (SPs) are underlined. The relation of modules to exons of the MtNOD25 gene isolated from cv. Jemalong is indicated, including two pseudoexons (PE1 and PE2) that correspond to modules found only in TC115445, probably due to differences in the exon/intron splice sites in the two cultivars. B, Modules of nodulin-25 proteins. Residues identical in at least $50 \%$ of the sequences are marked in bold and the SP predicted by Signal P (Emanuelsson et al. 2007) is underlined. C, Comparison of the C-termini of modular nodulins from $M$. truncatula (MtNOD25), M. sativa (MsNOD25) (Kiss et al. 1990a), Vicia faba (VfNOD32a) (Küster et al. 1994), and Galega orientalis (GOCA-04) (Kaijalainen et al. 2002).
In recent years, proteomics experiments revealed that some 200 legume proteins are specifically translocated to either the PBS or PBM (Catalano et al. 2004; Saalbach et al. 2002; Wienkoop and Saalbach 2003). To ensure the correct targeting of these proteins, three major pathways were proposed by Catalano and associates (2004), two of them relying on N-terminal signal peptides (SP) that mediate either cotranslational or posttranslational protein translocation. In their study, Catalano and associates (2004) reported that M. truncatula modular nodulins (nodulin-25, encoded by the MtNOD25 gene) are associated with the inner side of the PBM, adding evidence to an earlier hypothesis by Kiss and associates (1990a) that these proteins are located at the symbiotic interface.

Recently, Liu and associates (2006) identified the calmodulin-like peribacteroid proteins MtCaML1-6. The corresponding genes were located in the vicinity of the nodulin genes MtN22 (Gamas et al. 1996) and MtNOD25, leading to the hypothesis that a conserved nodule-specific 5 ' sequence encoding an SP was translocated to MtCaML1-6 via exon shuffling (Liu et al. 2006). The authors also found that homologous $5^{\prime}$ regions existed in the $M$. sativa nodulin gene $\mathrm{Nms}$ 32/34, and in four Lotus japonicus and two Lupinus luteus transcript sequences (Ganter et al. 1998; Liu et al. 2006; Swiderski et al. 2000).

Due to their proposed peribacteroid localization, an elucidation of the subcellular translocation of modular nodulins was of interest in order to improve the understanding of protein targeting to symbiosomes. Together with an in situ expression study of MtNOD25 in root nodules, we here present the subcellular localization of MtNOD25-mGFP6 translational fusions in the infected cells. Our results indicate that, similar to the MtCaML proteins (Liu et al. 2006), nodulin-25 is specifically translocated to the symbiosomes. Moreover, we demonstrate that the N-terminal SP of MtNOD25 found to be conserved across different symbiosome proteins is indeed sufficient for this subcellular targeting. This finding on the one hand sheds light on one possible mechanism for protein targeting in the infected cells of root nodules and on the other hand provides the first experimental evidence for a short molecular-address label capable of specifically directing legume proteins into the vicinity of nitrogen-fixing bacteroids.

\section{RESULTS AND DISCUSSION}

\section{Properties of the MtNOD25 gene encoding modular nodulins of $M$. truncatula.}

Three different MtNOD25 transcript sequences encoding modular nodulins are listed in the Dana Farber Cancer Institute (DFCI) M. truncatula Gene Index 9. Similar to modular nodulins from $V$. faba, V. hirsuta, and $M$. sativa (Küster et al. 1994, 1999; Kiss et al. 1990a), MtNOD25 proteins consist of different repetitive modules flanked by distinct $\mathrm{N}$ - and $\mathrm{C}$-termini (Fig. 1A and B). The altered number of repetitive modules in MtNOD25 cDNAs (Fig. 1A) indicates the existence of splice variants, reminiscent of the situation in Vicia spp. (Küster et al. 1999). Whereas only limited homologies exist between repetitive sequences of modular nodulins from different legumes (data not shown), the C-terminal modules are highly similar (Fig. 1C), indicating a conserved function. Based on SignalP (Emanuelsson et al. 2007), the N-terminus of MtNOD25 contains an SP with a cleavage site between amino acids 24 and 25 (Fig. 1A and B).

Nodule expressed sequence tags (EST) encoding modular nodulins were found in Medicago, Galega, and Vicia spp., all belonging to the galegoid legumes (Doyle and Luckow 2003) but not in the phaseoloid legumes Glycine max and Lotus japonicus. To infer putative functions for modular nodulins, we 
performed sequence- and motif-based homology searches against the current releases of GenBank, Swissprot (Bairoch et al. 2004), Uniprot (Boutet et al. 2007), and Interpro (Quevillon et al. 2005); however, these searches identified no significantly homologous sequences from nonlegumes. This indicates that modular nodulins are legume-specific proteins (Graham et al. 2004) and, although 5' gene sequences encoding MtNOD25like N-terminal SP are present in legumes forming determinate nodules (Liu et al. 2006), are characteristic of those nodules where bacteroids undergo terminal differentiation. In these nodules, other legume-specific proteins (e.g., cysteine-cluster and glycine-rich proteins) were proposed to play a role during the terminal differentiation of bacteroids (Alunni et al. 2007; Frühling et al. 2000; Mergaert et al. 2003).

\section{The MtNOD25 gene is located in a symbiosis island.}

In the current $M$. truncatula genome release 2.0 , an incomplete MtNOD25 sequence is present on BAC MtH2-124L21. To clone the complete MtNOD25 gene, overlapping BACs (Nam et al. 1999) were identified using cDNA probes. Two of these BACs contained a 10.5-kb EcoRI fragment that was also observed in genomic Southern hybridizations (Supplementary Fig. S1). In addition to the complete sequence of this fragment (AJ277858, Supplementary Fig. S2), the terminal sequences of four MtNOD25 BACs (AJ311512 to AJ311519) were obtained. BAC MtH2-124L21 partially matched the sequence of the 10.5-kb EcoRI fragment, indicating that MtNOD25 maps at $\approx 34$ centimorgans on chromosome III (Thoquet et al. 2002). To the left of MtNOD25, this contig contained the nodule-specific gene MtN22 (Gamas et al. 1996) and the MtCaML6 gene (Fedorova et al. 2002) encoding a nodule-specific calmodulinlike protein. The identification of MtN22 and MtCaML6 next to MtNOD25 confirms the report of Liu et al. (2006) that MtNOD25 is located downstream of MtN22 and a family of six $M t C a M L$ genes, in a genomic region discussed as a symbiosis island (Zhu et al. 2003).

The general exon/intron structure of MtNOD25 is similar to that of the MsNOD25 gene (Végh et al. 1990) (Supplementary Fig. S3). Interestingly, a repetitive module missing in several MtNOD25 transcripts (Fig. 1A) corresponds to exon E7. This finding confirms that alternative splicing can generate different MtNOD25 variants. In addition to the 13 MtNOD25 exons covered by EST, a pseudoexon (PE1, position 7,428 to 7,481) has a typical MtNOD25 exon sequence but is probably not included in M. truncatula cv. Jemalong MtNOD25 transcripts due to an altered 5' splice site.

\section{The MtNOD25 gene is exclusively expressed in the infected cells of $M$. truncatula nodules.}

MtNOD25 gene expression was determined by real-time reverse-transcription polymerase chain reaction (RT-PCR) in young and mature root nodules, arbuscular mycorrhizal (AM) roots, uninfected roots, leaves, flowers, and pods. These experiments revealed exclusive MtNOD25 expression in nodules, whereas the gene was not induced in AM roots or nonsymbiotic tissues (data not shown). The strictly nodule-specific expression is supported by the fact that more than $100 \mathrm{MtNOD} 25$ EST from the DFCI M. truncatula Gene Index 9 (Quackenbush et al. 2001) were exclusively derived from nodules or nodulated roots. In addition, the MtNOD25 promoter sequence was similar to the promoter sequence of the VfNOD28/32 gene that was specifically active in the central region of root nodules (Fig. 2) (Küster et al. 1999).

To monitor the accumulation of MtNOD25 transcripts within nodule tissues, in situ hybridizations were carried out using $M$. truncatula root nodules at 25 days postinoculation (dpi) with Sinorhizobium meliloti. In longitudinal sections
(Fig. 3A and B), enhanced MtNOD25 transcript levels occurred in the infected cells of the nitrogen-fixing zone, whereas control hybridizations with a sense probe did not exhibit comparable staining (data not shown).

Preceding the MtNOD25 gene, a 1,462-bp promoter region contained a motif resembling the organ-specific element (OSE) of leghemoglobin gene promoters. We narrowed down the MtNOD25 promoter sequences mediating nodule-specific expression by the use of promoter-gusAint fusions expressed in transgenic $M$. truncatula nodules. Histochemical analyses showed that deletions up to position $-1,106$ did not affect strong and specific expression in the infected cells of root nodules (Fig. 3C and D), similar to the activity of the MsNOD25 promoter (Kiss et al. 1990b). Deletions down to position -299 (Fig. 3E and F) had no qualitative effect on gene expression, although the expression intensity was somewhat reduced. Furthermore, deletion -299 of the MtNOD25 promoter lost its exclusive expression in the infected cells, because it displayed a residual activity in the nodule vasculature (Fig. 3F). A further deletion up to position -101 removed the $5^{\prime}$ portion of the OSE and abolished promoter activity completely (data not shown), indicating that the major control elements driving gene expression in the infected cells reside between positions -101 and -299 . This is in line with a high sequence identity between modular nodulin gene promoters from $V$. faba and $M$. truncatula in this area (Fig. 2) and matches the observation that MtCaML1 and MtCaML2 sequences are similar to MtNOD25 promoters in this region as well (Liu et al. 2006). Nevertheless, upstream sequences contribute to expression intensity and to an exclusive MtNOD25 expression in the infected cells. This resembles the situation in soybean nodulin genes, where OSE require the presence of upstream activating sequences to drive gene expression to the infected cells of root nodules (Stougaard et al. 1990).

\section{MtNOD25-mGFP6 fusions are targeted to the symbiosomes.}

To investigate the subcellular localization of MtNOD25 in the infected cells of root nodules, different translational fusions to the $m$ GFP6 reporter gene were constructed (Fig. 4) and their expression was monitored in transgenic $M$. truncatula nodules (Fig. 5). Because the Cauliflower mosaic virus (CaMV) $35 \mathrm{~S}$ promoter is primarily expressed in the uninfected cells of root nodules and, thus, is not well suited for studying the localization of translational fusions in the infected cells of central nodule tissues (Auriac and Timmers 2007), we have chosen the

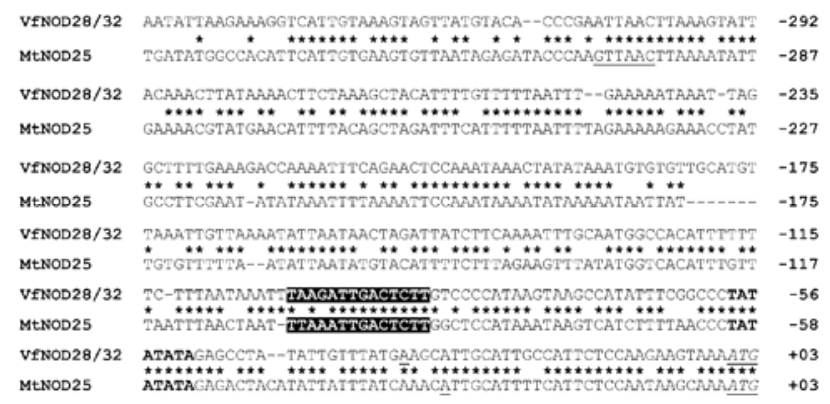

Fig. 2. Comparison of the immediate upstream promoter regions of VfNOD28/32 and MtNOD25. Identical positions are marked by asterisks. A region resembling organ-specific elements of leghemoglobin and other nodulin gene promoters is shown inverted, whereas putative TATA-boxes are marked in bold. The transcriptional start site of VfNOD28/32 (Küster et al. 1999) and the most $5^{\prime}$ residues of MtNOD25 cDNAs (TC113614) are underlined, whereas the ATG start codons are additionally shown in italics. An HpaI site defining the extent of the -299 deletion of the MtNOD25 promoter is underlined. 
native $M t N O D 25$ promoter $(-1,072 /-5)$ to drive expression of MtNOD25-mGFP6 fusions (Fig. 4). An inspection of transgenic $p N 25-N 25$-green fluorescent protein $(G F P)$ nodules colonized by an $S$. meliloti strain expressing a constitutive $\mathrm{m}$ red fluorescent protein (RFP) (Smit et al. 2005) revealed co-

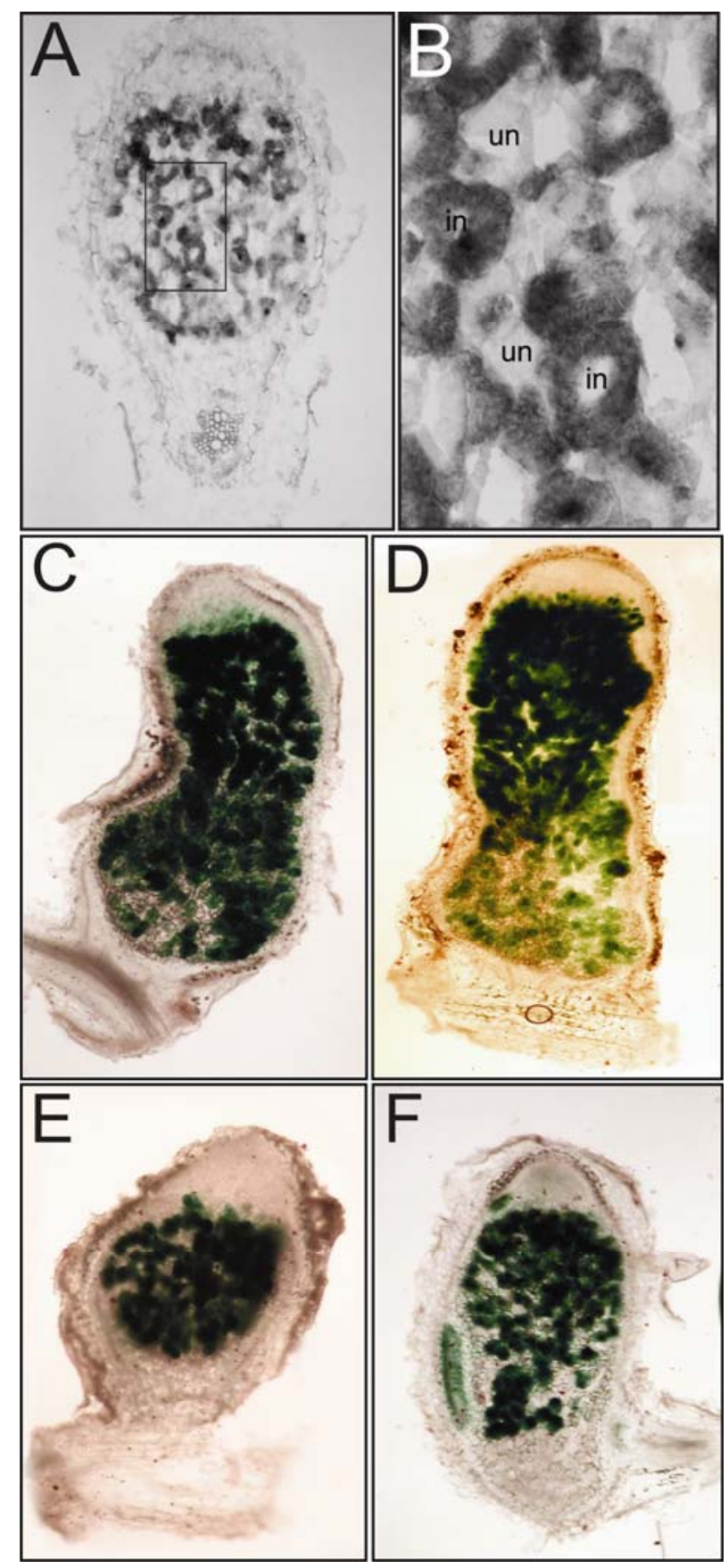

Fig. 3. Cellular expression of the MtNOD25 gene in Medicago truncatula root nodules. A and B, Localization of MtNOD25 transcripts in M. truncatula root nodules. A, Longitudinal cross section of a mature $M$. truncatula root nodule hybridized against an MtNOD25 antisense target. Highest expression is found in the infected cells throughout the nitrogen-fixing zone III, whereas control hybridizations with a sense probe did not exhibit any comparable staining (data not shown). B, Magnification of the area boxed in A. MtNOD25 transcripts are detected only in the infected cells. C through F, Activity of pMtNOD25-gus Aint fusions in sections of transgenic $M$. truncatula root nodules: C, $-1,462 /-4$; D, $-1,106 /-4$; E, $-436 /-4$; and F, -299/-4 promoter regions. Except for the -299/-4 pMtNOD25gusAint fusion displaying weak activity in the nodule vasculature, strong and specific promoter activity was detected in the infected cells of the central nodule tissue. Abbreviations: in, infected cells; un, uninfected cells. localization of mGFP and mRFP in the infected cells of root nodules (Fig. 5A), with no leakage of the fusion proteins to the uninfected cells being evident (Fig. 5A and B). Subcellular GFP fluorescence was observed in distinct circular and sicklelike structures surrounding the bacteroids (Fig. 5C), whereas the vacuole, nucleus, cell membrane, and cell wall of the infected cells did not display GFP fluorescence (Fig. 5B). Together, the analysis of GFP fluorescence in transgenic root nodules indicates an exclusive localization of MtNOD25mGFP6 fusion proteins in the infected cells (Fig. 5A through $\mathrm{C})$, in accordance with the expression pattern of the MtNOD25 promoter (Fig. 3). On the subcellular level, our data demonstrate that MtNOD25-mGFP6 fusions are efficiently and specifically targeted to the symbiosomes in the infected cells of root nodules. Together with the MtCaML protein family described by Liu and associates (2006) and the ENOD8 protein encoding a nodule-specific esterase (Coque et al. 2008), modular nodulins are among the few known legume proteins that are approved to pass through the PBM to accumulate in the PBS, thus contributing to a specific protein composition of the symbiosome.

\section{The MtNOD25 signal peptide targets mGFP6 proteins} to the symbiosome.

The N-terminal module of MtNOD25 largely consists of an SP (Fig. 1) with a proposed cleavage site between amino acids 24 and 25 (SignalP-score 0.98) (Emanuelsson et al. 2007). On the structural level, this SP consists of a characteristic basic nregion and a classical hydrophobic h-region as well as a typical polar c-region, and its amino acid sequence conforms to the $-3 /-1$ rule defined by von Heijne (1986). Together, these properties result in an SP probability of 0.99 and a signal anchor probability of only 0.01 according to SignalP (Emanuelsson et al. 2007), allowing the conclusion that the MtNOD25 SP is most probably cleaved and does not function to anchor MtNOD25 in the PBM.

Significant homologies of the N-terminal MtNOD25 module exist not only to N-termini of six $M$. truncatula genes encoding nodule-specific calmodulin-like proteins (MtCaML), the M. truncatula nodulin gene MtN22, and the M. sativa nodulin gene Nms-32/34, but also to Lupinus luteus and Lotus japonicus EST (Ganter et al. 1998; Liu et al. 2006; Swiderski et al. 2000). These similarities in all cases are confined to the Nterminal region belonging to the predicted SP (Fig. 6A), with sequence identities or similarities declining immediately after the SP cleavage site proposed by SignalP (Emanuelsson et al. 2007). This finding allowed us to define a characteristic MtNOD25-like SP motif visualized in Figure 6B. Similar to nodulin-25 (discussed above), all other proteins containing an

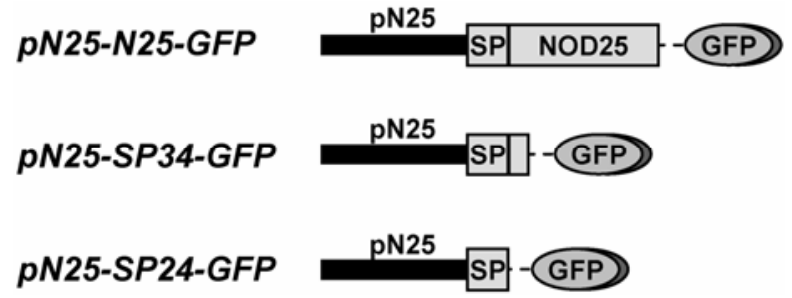

Fig. 4. Fusions of defined regions of the MtNOD25 protein to mGFP6 The complete MtNOD25 coding region (pN25-N25-GFP), the MtNOD25 N-module (pN25-SP34-GFP) region, and the MtNOD25 (pN25-SP24GFP) region were fused to the $m G F P 6$ reporter gene. Expression of all fusions is mediated by the MtNOD25 promoter fragment from position $-1,072$ to -5 (relative to the start codon). A dotted line denotes an amino acid linker. Abbreviations: SP, signal peptide-encoding MtNOD25 region; $G F P$, coding region for the green fluorescent protein; $\mathrm{pN} 25$, promoter of the MtNOD25 gene. 
N-terminal MtNOD25-like SP have a high SP probability and a low signal anchor probability according to SignalP (data not shown) (Emanuelsson et al. 2007). The remaining protein sequences of MtCaML1-6, MtN22, and all other proteins containing an MtNOD25-like SP motif (Fig. 6A) displayed no similarities to MtNOD25, and no SP from any other known protein displayed a comparable similarity to the MtNOD25 SP; therefore, it is likely that the SP itself contains sufficient information for protein targeting to symbiosomes.

To test whether the MtNOD25 SP is able to accumulate mGFP6 proteins in the symbiosome as does the full-size MtNOD25mGFP construct presented above, we fused two regions deriving from the $\mathrm{N}$-terminal MtNOD25 module to the mGFP6 reporter gene (Fig. 4). Including the start codon, the encoded N-terminal peptides consisted of the first 34 and 24 amino acids of MtNOD25, respectively, followed by a GGRSPGGS linker (discussed below) and the $m G F P 6$ coding region, resulting in constructs $p N 25-S P 34-G F P$ and $p N 25$ $S P 24-G F P$, respectively. Because the sequence of the SP was not altered, these fusions were all predicted to be cleaved between amino acids 24 and 25 (SignalP) (Emanuelsson et al. 2007).

When analyzing nodule cells colonized by an $S$. meliloti strain expressing a constitutive mRFP protein (Smit et al. 2005), we observed the same pattern of GFP expression as detailed above for the full-size $p N 25-N 25-G F P$ fusion, regardless of the presence of 34 (Fig. 5D) or only 24 amino acids preceding the mGFP6 sequence (Fig. 5E and $\mathrm{G}$ through I, respectively). Again, GFP fluorescence closely and completely surrounded the space occupied by the bacteroids (Fig. 5D and E) in the infected cells, visualized via co-localization with red fluorescent $S$. meliloti (Fig. 5I). This GFP pattern excluded a translocation to the bacteroids and indicated an accumulation of MtNOD25mGFP6 fusion proteins in the PBS. Most importantly, GFP fluorescence was observed throughout the apparent lumen of the peribacteroid space, with no visible space between GFP fluorescence and mRFP-labeled bacteroids. This observation confirms that the $\mathrm{N}$-terminal presence of an original 24-amino-acid MtNOD25 SP is sufficient to direct specific GFP fluorescence to the symbiosomes of infected root nodule cells.
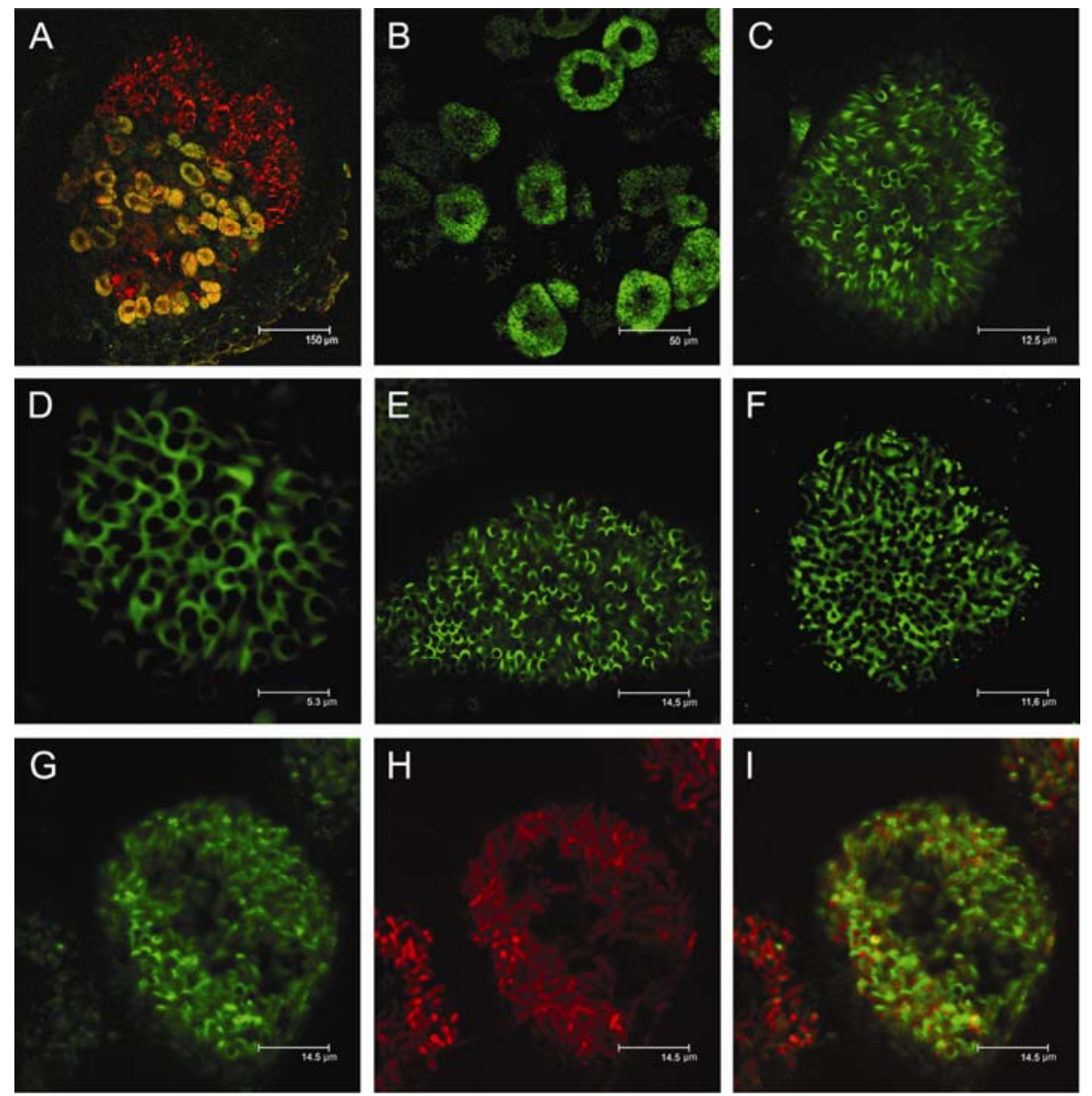

Fig. 5. Confocal imaging of MtNOD25-mGFP6 fluorescence in transgenic root nodules. A through C, Green fluorescent protein (GFP) fluorescence in a transgenic nodule expressing a pN25-N25-GFP translational fusion. A, Merged picture of GFP and m-red fluorescent protein (RFP) of a longitudinal nodule section colonized with S. meliloti strain 2011-mRFP. GFP is detected in the infected cells of the central nodule tissue. Red fluorescence is detected in the prefixing-zone, interzone II-III, infection threads, and infected cells of the nitrogen-fixing zone III (Vasse et al. 1990). Note that the yellow coloration in the infected cells occurs only when the signal intensity of both fluorophores is located at the same scan layer. No co-localization is visible in the prefixing zone. B, GFP localization in the central nodule tissue showing exclusive GFP detection within the infected cells. C, Close-up of a single infected cell showing circular and sickle-like GFP signals. D, Localization of GFP fluorescence in an infected cell expressing the $p N 25$-SP34-GFP fusion. E, Localization of GFP fluorescence in an infected cell expressing the $p N 25-S P 24-G F P$ fusion. F, Localization of GFP fluorescence in an infected cell expressing the $p N 25-S P 34-$ GFP fusion upon colonization with the fix ${ }^{-}$mutant strain 1021 nifH::Tn5. G through I, Localization of GFP and mRFP in an infected cell expressing the pN25-SP24-GFP fusion upon colonization with the red fluorescent S. meliloti strain Sm2011-mRFP. G, GFP localization. H, mRFP localization of the same layer shown in G. The mRFP signal uncovers the presence and density of bacteroids within the infected cell. I, Merged picture of the mGFP6 image (G) and the mRFP image $(\mathrm{H})$. The bacteroids are closely surrounded by the GFP signal. Image B is a superimposed image based on three optical sections. All other images represent single optical sections. Scale bars are indicated. 
To prove whether the NOD25 protein localization in the symbiosome is depended on active nitrogen fixation, we used the $S$. meliloti mutant Sm1021 nifH::Tn5 to nodulate $M$. truncatula. In fix root nodules, GFP fluorescence was restricted to a group of cells immediately below the interzone II-III region (data not shown) and, here, the symbiosome localization of GFP was comparable with the situation in root nodules colonized by wild-type rhizobia (Fig. 5F). The absence of fluorescence in regions of root nodules with more mature infected cells (data not shown) indicates that the MtNOD25 promoter is inactive in the absence of symbiotic nitrogen fixation, adding evidence that the regulation and function of nodulin-25 is tightly connected to the nitrogen fixation process.

\section{Possible routes for the symbiosome targeting of MtNOD25 proteins.}

Searches of the available $M$. truncatula genome sequence and the DFCI $M$. truncatula Gene Index did not result in the identification of proteins containing MtNOD25-like signal peptides in addition to MtCaML1-6 and MtN22 (Fig. 6), indicating that the mode of symbiosome targeting by MtNOD25like signal peptides cannot account for the $\approx 200$ proteins that have been identified in this compartment by proteomics

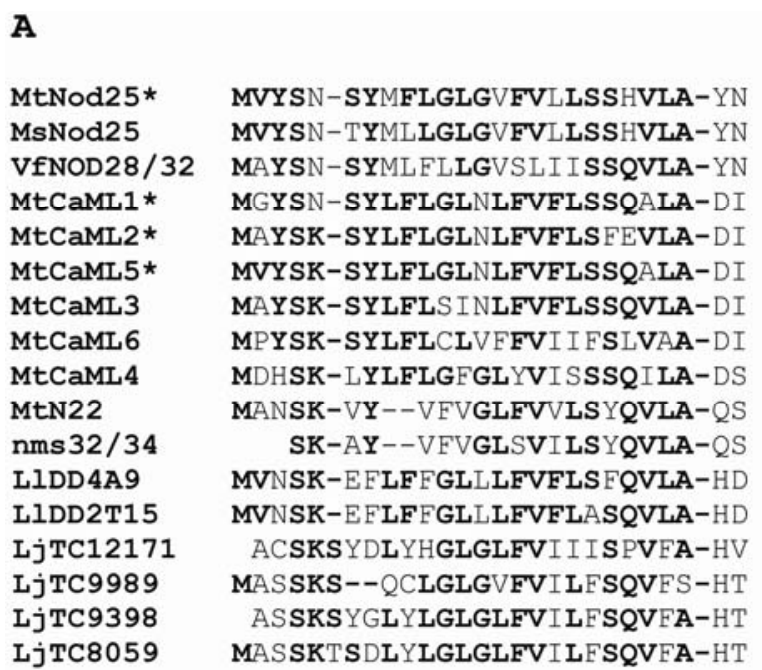

B

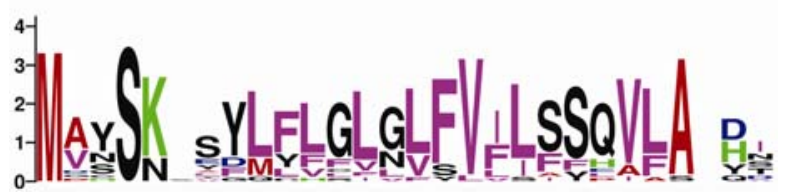

Fig. 6. Comparison of signal peptides from different nodule-specific proteins. A, Comparison of the N-terminal signal peptides (SPs) of the modular nodulins MtNOD25, MsNOD25 (Kiss et al. 1990a), and VfNOD28/32 (Küster et al. 1994) to the SPs of the Medicago truncatula MtCaML1-6 proteins (MtCaML1, DFCI Mt Gene Index TC134855; MtCaML2, TC114830; MtCaML5, TC125585; MtCaML3, TC114830; MtCaML6, TC118427; MtCaML4, TC114792) (Liu et al. 2006), M. truncatula nodulin MtN22 (Gamas et al. 1996), M. sativa nodulin nms32/34 (Ganter et al. 1998), Lotus japonicus nodulins encoded by cDNAs TC12171, TC9989, TC9398, and TC8059 (Liu et al. 2006), as well as the lupin nodulins L1DD4A9 and L1DD2T15 (Swiderski et al. 2000). Prediction was done using SignalP (Emanuelsson et al. 2007), and the first 24 amino acids up to the predicted cleavage site are shown. The most frequent amino acids are shown in bold. Proteins located in the symbiosome by proteomics (Catalano et al. 2004) or GFP fusions (this work, Liu et al. 2006) are marked by asterisks. B, Sequence logo of the alignment shown in A (Crooks et al. 2004).
(Catalano et al. 2004; Saalbach et al. 2002; Wienkoop and Saalbach 2003). Thus, it is possible that a special targeting mechanism is used by MtNOD25-like SP. Theoretically, symbiosome targeting can occur post-translationally, subsequent to protein synthesis on cytoplasmic ribosomes, or co-translationally, via synthesis into the lumen of the rER and subsequent vesicle transport to symbiosomes (pathway I and II/III, respectively; according to Catalano and associates [2004]). Assuming that the MtNOD25 SP is indeed cleaved by a signal peptidase upon translocation into the rER, the subsequent specific accumulation of mGFP6 in the symbiosome would require that a targeting via rER or Golgi complexes operates independently of the SP, possibly via specific glycosylation or another decoration of the remaining amino acid sequence. In case of the fusion of only the 24 amino acids of the N-terminal MtNOD25 SP to $m G F P 6$, such a modification could only reside in the GFP sequence itself; however, cytoplasmic GFP proteins are not known to be translocated across endomembranes without the presence of an N-terminal SP. Thus, it is possible that the $\mathrm{N}$-terminal MtNOD25 SP is cleaved later during co-translational PBS targeting and, thus, could still function as an $\mathrm{N}$-terminal targeting signal during the passage via the rER and the Golgi complex.

Because such a delayed cleavage of SP would be unusual (Nothwehr and Gordon 1990), it appears more likely that MtNOD25-like SP operate post-translationally. One example for a post-translational SP targeting in plants is given by Chen and associates (2004). Using transgenic tobacco and rice protoplasts, the authors demonstrated that the rice $\alpha$-amylase ( $\alpha$ Amy3) is only transferred into both chloroplasts and extracellular compartments when it contains its SP, whereas $\alpha A m y 3 \Delta S P$, missing the SP, remains in the cytoplasm. The authors could show that the SP alone was sufficient to mediate a specific translocation of GFP into chloroplasts (Chen et al. 2004). Similar to this process, symbiosome translocation of MtNOD25 apparently exclusively requires its N-terminal SP. Thus, it could be assumed that this SP directly enters the symbiosome membrane instead of the rER and, upon this, would directly be cleaved at the site of cargo destination. Catalano and associates (2004) had identified MtNOD25 proteins as components of the PBM fraction but, as stated by the authors, this co-purification might have resulted from an association of MtNOD25 with the inner PBM layer and, thus, does not exclude a PBS localization. Although the spatial resolution in fluorescence microscopy is limited, our subcellular localizations-in addition to the molecular properties of the MtNOD25 SP detailed above-are in favor of a release of MtNOD25 in the lumen of the PBS.

Together, our results elucidate one of several molecular mechanism for protein targeting to nitrogen-fixing symbiosomes by defining a short, 24-amino-acid address label. All other modules of MtNOD25, including the C-terminus that is particularly conserved across modular nodulins from different legumes (Fig. 1), are not involved in symbiosome targeting and, thus, are probably linked to the so far elusive function of MtNOD25 proteins.

\section{Conclusions and perspectives.}

In addition to the genes discussed here, large families of glycine-rich protein (GRP) and cysteine-cluster protein (NCR) genes are exclusively present in nodules where terminal bacteroid differentiation occurs, and it was proposed that the NCR and GRP proteins might be involved in controlling this differentiation process (Alunni et al. 2007; Mergaert et al. 2003). Due to their specific symbiosome localization, a possible function of MtNOD25 proteins might also be related to the fact that bacteroids differentiate over time in M. truncatula root 
nodules. Interestingly, the sequences of MtNOD25-like SP are clearly different from those of the GRP and NCR proteins as well as the ENOD8 esterase (Alunni et al. 2007; Coque et al. 2008; Mergaert et al. 2003). Thus, it remains to be elucidated whether SP with different primary sequences can also mediate symbiosome targeting on their own or whether other targeting signals are involved. Finally, our results not only contribute to the identification of molecular mechanisms facilitating symbiosome targeting but also define a short address label that can be used as a molecular tool to target proteins or peptides to the symbiosomes in order to engineer the protein composition of the PBS or to deliver peptides that influence the metabolism of nitrogen-fixing bacteroids or the signal exchange between both symbionts.

\section{MATERIALS AND METHODS}

Plants, bacterial strains, libraries, recombinant DNA techniques, and DNA sequencing.

Seed of wild type $M$. truncatula Jemalong A17 were surface sterilized, scarified, and vernalized as described by Hohnjec and associates (2003). After germination, plants were grown in washed and sterilized clay granulate (Seramis, Masterfoods $\mathrm{GmbH}$, Verden, Germany) using S. meliloti 1021 (Meade and Signer 1977) or Glomus intraradices (Premier Tech Biotechnologies, Riviere-du-Loup, Canada) for nodulation and mycorrhization, respectively, as described previously (Hohnjec et al. 2003).

To visualize $S$. meliloti inside root nodules, strain Sm2011mRFP expressing a monomeric red fluorescent protein (Smit et al. 2005) was used. Plants harvested for in situ hybridizations were nodulated with $S$. meliloti RCR2011 pXLGD4 (Journet et al. 2002), and nodules were collected at 25 dpi. Strain Sm1021 nifH::Tn5 (provided by A. Becker, Institute for Genome Research and Systems Biology, Bielefeld University, Germany) was used to induce fix root nodules.

M. truncatula cv. Jemalong J5 (Gamas et al. 1996) and M. truncatula cv. R108 (Györgyey et al. 2000) nodule cDNA libraries were used to isolate full-length cDNAs. Filters representing a genomic BAC library of $M$. truncatula cv. Jemalong A17 (Nam et al. 1999) were obtained from D. Cook (University of California-Davis, Davis, U.S.A.). Screening of cDNA and genomic libraries was performed using standard protocols (Sambrook et al. 1989).

All in vitro DNA manipulations and cloning experiments were carried out in Escherichia coli XL1Blue (Bullock et al. 1987) using pK18 (Pridmore 1987) or pBluescript (Stratagene Europe, Amsterdam, The Netherlands) plasmids. Plasmid DNA was prepared using the Plasmid Mini Kit (Qiagen, Hilden, Germany), and in vitro manipulations of DNA were performed using standard protocols (Sambrook et al. 1989). DNA sequencing was performed on an ABI PRISM 377 DNA Sequencer (Applied Biosystems, Darmstadt, Germany).

\section{Identification of full-length \\ MtNOD25 transcript sequences.}

Using an MtNOD25 RT-PCR product from M. truncatula nodule RNA, full-length MtNOD25 transcript sequences were isolated from root nodule cDNA libraries of $M$. truncatula cv. R108 (Györgyey et al. 2000) and M. truncatula cv. Jemalong J5 (Gamas et al. 1996). Sequence analysis revealed that cDNA MtNOD25-3 (GenBank accession AJ308588) from M. truncatula cv. R108 as well as cDNAs MtNOD25 -213, -12, and -25 (AJ277859, AJ277860, and AJ277861) from M. truncatula cv. Jemalong J5 represented complete or near-complete MtNOD25 coding regions.

In the DFCI Medicago truncatula Gene Index 9, three tentative consensus sequences (TC) cover complete or near-com- plete MtNOD25 sequences (Fig. 1A). Specifically, cDNA MtNOD25-3 is part of TC115445, cDNAs MtNOD25-25 and 213 are assembled in TC113614, and cDNA MtNOD25-12 contributes to TC119488. Whereas TC113614 and TC119488 are based only on EST from $M$. truncatula cv. Jemalong, TC115455 was assembled from $M$. truncatula cv. R108 EST. Additional MtNOD25 sequences were chimeric or contained only partial MtNOD25 coding regions that were identical to the three TC described above. Because they did not reveal any additional information, they were not considered further.

\section{Southern hybridization.}

Southern hybridizations were carried out using $\approx 10 \mu \mathrm{g}$ of genomic $M$. truncatula cv. Jemalong A17 DNA or $\approx 1 \mu \mathrm{g}$ of BAC DNA digested with appropriate restriction enzymes, respectively. Digests were separated on $1 \%$ (wt/vol) agarose gels and vacuum blotted onto nylon membranes (Roche Diagnostics $\mathrm{GmbH}$, Mannheim, Germany). Hybridizations were carried out at $42^{\circ} \mathrm{C}$ in DIG Easy Hyb solution (Roche Diagnostics $\mathrm{GmbH}$ ) using digoxigenin (DIG)-labeled probes (PCR DIG Probe Synthesis Kit; Roche Diagnostics $\mathrm{GmbH}$ ). Washes were carried out at $65^{\circ} \mathrm{C}$ in $2 \times \mathrm{SSC}(1 \times \mathrm{SSC}$ is $0.15 \mathrm{M} \mathrm{NaCl}$ plus $0.015 \mathrm{M}$ sodium citrate) and $0.1 \%$ (wt/vol) sodium dodecyl sulfate (SDS) for $5 \mathrm{~min}$ and in $0.5 \times \mathrm{SSC}$ and $0.1 \%$ (wt/vol) SDS for $15 \mathrm{~min}$. Detections were carried out using the DIG Luminescent Detection Kit (Roche Diagnostics GmbH).

\section{Real-time RT-PCR experiments.}

Total RNA was isolated using the RNeasy Plant Mini Kit in conjunction with DNaseI treatment (Qiagen). Real-time RTPCR experiments were performed as reported previously (Hohnjec et al. 2005) using primers specific for MtNOD25 (TGTTGAATCACTTAACTTAGG and TAAGCAAAATGGT TTATTCAA) and MtTef-lalpha (Hohnjec et al. 2003). Realtime RT-PCR products were scored as detectable if they were amplified after less than 40 PCR cycles.

\section{In situ hybridizations.}

DIG-labeled sense and antisense riboprobes of MtNOD25 were obtained from a linearized plasmid containing a fulllength MtNOD25 cDNA using the DIG RNA labeling Kit (Spe6/T7; Roche Diagnostics GmbH). Subsequent to DIGlabeling, alkaline hydrolization delivered labeled RNA fragments of $\approx 150$ bases. For embedding in paraffin, nodules were fixed in $4 \%$ (wt/vol) paraformaldehyde and $0.1 \%$ (vol/vol) Triton X-100 in phosphate-buffered saline following the protocol of Schaarschmidt and associates (2006). Embedded nodules were cut in median longitudinal sections of $8 \mu \mathrm{m}$ using a rotary microtome (HM355; Microm International, Walldorf, Germany). Sections were mounted onto slides coated with poly-L-lysine (Sigma-Aldrich), and the hybridization procedure was performed as described by Schaarschmidt and associates 2006. Micrographs were taken using a Zeiss Axioplan microscope (Carl Zeiss Jena GmbH, Jena, Germany) equipped with a video camera (Fujix Digital Camera HC-300Z; Fuji Photo Film, Tokyo) and were processed via Photoshop 7.0 (Adobe Systems, San Jose, CA, U.S.A.).

\section{Constructing gusAint fusions of promoter regions and histological analysis of transgenic nodules.}

The MtNOD25 promoter was PCR amplified from a pK18 plasmid carrying a 10.581 -bp genomic MtNOD25 EcoRI fragment (GenBank accession AJ277858), using the M13 reverse primer and a primer that introduced a HindIII site immediately following position -8 of the MtNOD25 promoter. Parts of the amplified native promoter regions were cloned as EcoRI/HindIII (-1,462/-4), NsiI/HindIII (-1,106/-4), StuI/HindIII (-436/-4), 
HpaI/HindIII (-299/-4), and SwaI/HindIII (-101/-4) fragments (positions relative to the start codon of the MtNOD25 gene) in front of the gusAint gene in plasmid pGUS-INT (Küster et al. 1995), making use of the HindIII site next to the gusAint start codon. All gusAint fusions were cloned as SpeI fragments into the $X b a \mathrm{I}$ site of plasmid pIV2 (Küster et al. 1995). All pIV2 derivatives were electroporated into Agrobacterium rhizogenes ARqual (Quandt et al. 1993), where they integrated into the $\mathrm{T}_{\mathrm{L}}$ DNA by a single crossover. A. rhizogenes strains were used to generate transgenic roots on $M$. truncatula cv. Jemalong A17 according to Vieweg and associates (2004). Between 2 and 3 weeks postinoculation with $S$. meliloti 1021, transgenic nodules were sectioned and stained for $\beta$-glucuronidase activity as reported by Hohnjec and associates (2003). Stained sections were examined by light microscopy and documented with an Olympus C-4040Z (Olympus, Hamburg, Germany) digital camera.

\section{Construction of MtNOD25-mGFP6 fusions and expression in planta.}

We used a derivative of the p35S-GFP vector (GenBank accession number U28417) (Clontech Laboratories, Palo Alto, CA, U.S.A.). An mGFP6 (Haseloff 1999; Haseloff et al. 1997) coding sequence was PCR amplified (AAAGGATCCATGCA TAAAGGAGAAGAACTTTTCACTGG and AAAGAGCTCT CACCCATCCTTTTTGTATAGTTCATCCAT) and introduced as BamHI/SacI fragment into p35S-GFP to replace the original GFP, resulting in plasmid p35S-mGFP6 (Holger Densow, Biometra GmbH, Göttingen, Germany).

Furthermore, we introduced an XbaI/BamHI (TCTAGAGG TACCAGATCTCCCGGGGGATCC) linker between the original $\mathrm{XbaI}$ and BamHI sites of p35S-mGFP6 to expand the mcs in front of the $m G F P 6$ coding region in $\mathrm{p} 35 \mathrm{~S}$-mGFP6, resulting in vector $\mathrm{p} 35 \mathrm{~S}$-OLI-mGFP6.

For an expression under native conditions, gene-specific primers extended with suitable $S p h \mathrm{I}$ and $K p n I$ restriction sites (AAAGCATGCGAATGATGATGAGGAAGAAA and AAA $\underline{G}$ GTACCCTTATTGGAGAATGAAAATG, respectively), were used to amplify an MtNOD25 promoter fragment from position $-1,072$ to -5 (relative to the start codon). Subsequently, we replaced the CaMV p35S promoter in p35S-OLI-mGFP6 by introducing the MtNOD25 promoter via the $S p h \mathrm{I} / K p n \mathrm{I}$ restriction sites to create vector pN25-OLI-mGFP6.

Gene-specific primers extended with suitable $K p n I$ or $B g l \mathrm{II}$ restriction sites (AAAGGTACCAAAAATGGTTTATTCAAAT TCA and AAAAGATCTTCCTCCTTCACTGGCAATATCATT, respectively) were used to amplify the complete MtNOD25 coding region (TC113614). During PCR, the three adenosine bases preceding the MtNOD25 start codon were conserved and the MtNOD25 reading frame was completely amplified up to and including the codon preceding the stop codon.

The MtNOD25 N-module derivatives SP34 and SP24 were also amplified, introducing suitable $K p n I$ and $B g l$ II restriction sites, respectively (AAAGGTACCAAAAATGGTTTATTCAA ATTCA, AAAAGATCTTCCTCCATGGATAGAGGGATCAG CACG and AAAGGTACCAAAAATGGTTTATTCAAATTCA, AAAAGATCTTCCTCCAGCCAAAACATGAGAAGAGAG), resulting in PCR products of 102 and $72 \mathrm{bp}$, respectively, of the native MtNOD25 sequence, commencing with the start codon. Again, three adenosine bases were retained in front of the ATG. To provide a sufficiently long amino acid linker between MtNOD25 and mGFP6 sequences, two codons for the amino acid glycine were introduced during PCR amplification. Together with the cloning site, this linker inserted a GGRSPGGS peptide linker between MtNOD25 and mGFP6. All cDNA derivatives were cloned in front of the $m G F P 6$ coding region in plasmid $\mathrm{pN} 25-\mathrm{OLI}-\mathrm{mGFP}$, resulting in the translational fusion $p N 25-N 25-G F P, p N 25-S P 34-G F P$, and $p N 25-S P 24-G F P$. The integrity of these fusions was verified by restriction analysis and DNA sequencing.

The $p N 25-N 25-G F P, p N 25-S P 34-G F P$, and $p N 25-S P 24-$ GFP fusions were cloned as EcoRI/HindIII fragments into the binary plasmid pBIN19 (Bevan 1984). The binary vector derivatives were electroporated into A. rhizogenes ARqua1 (Quandt et al. 1993) and the resulting strains were used to generate transgenic roots on $M$. truncatula cv. Jemalong A17 according to Quandt and associates (1993) on solid media containing $\mathrm{Kan}_{40}$ for selection of transgenic hairy roots. After 3 weeks, plants were transferred into open pots filled with clay granulate (Seramis, Masterfoods $\mathrm{GmbH}$, Verden, Germany) and nodulated with the microsymbiont as described above.

Transgenic $M$. truncatula roots were analyzed by confocal laser-scanning microscopy (CLSM). Semi-thin sections (80 $\mu \mathrm{m}$ ) of root nodules were obtained as described by Hohnjec and associates (2003) using a Leica VT $1000 \mathrm{~S}$ vibratome (Leica, Heidelberg, Germany). In vivo visualization of mGFP6 and mRFP (Smit et al. 2005) fluorophores was performed with a Leica TCS SP2 AOBS CLSM system equipped with an argon and a helium laser, respectively (emission at 488 and $543 \mathrm{~nm}$ ) (Leica, Heidelberg, Germany). Laser scanning was performed using settings for excitation and emission wavelengths at 488 and 500 to $535 \mathrm{~nm}$ and 543 and 600 to $630 \mathrm{~nm}$, respectively. Image processing was carried out with the Leica confocal software lite (version 2.61; Leica). For Figure 4B, a series of three optical sections, each with a $0.2-\mu \mathrm{m}$ interval on the $\mathrm{z}$-axis, was collected and superimposed to create an extended depth-of-focus image. To differentiate true GFP signals (peaking at $\approx 514 \mathrm{~nm}$ ) from autofluorescence, images were routinely verified by lambda scans.

\section{ACKNOWLEDGMENTS}

We are grateful to P. Gamas (INRA-CNRS, Toulouse, France) and M. Crespi (CNRS, Gif-sur-Yvette, France) for providing M. truncatula cDNA libraries, to T. Huguet (INRA-CNRS, Toulouse, France) for supplying $M$. truncatula Jemalong A17 seed, and to T. Bisseling (Wageningen University, The Netherlands) for the supply of $S$. meliloti Sm2011-mRFP. Nylon membranes representing a genomic BAC library of $M$. truncatula cv. A17 were a kind gift of D. Cook (University of California-Davis, Davis, U.S.A.). We acknowledge H. Densow (Biometra GmbH, Göttingen, Germany) for sharing the p35S-mGFP6 vector. N. Hohnjec and H. Küster received financial support from the "International Graduate School in Bioinformatics and Genome Research" (CeBiTec, Bielefeld University, Germany).

\section{LITERATURE CITED}

Alunni, B., Kevei, Z., Redondo-Nieto, M., Kondorosi, A., Mergaert, P., and Kondorosi, E. 2007. Genomic organization and evolutionary insights on GRP and NCR genes, two large nodule-specific gene families in Medicago truncatula. Mol. Plant-Microbe Interact. 20:1138-1148.

Auriac, M. C., and Timmers, A. C. 2007. Nodulation studies in the model legume Medicago truncatula: advantages of using the constitutive EF1alpha promoter and limitations in detecting fluorescent reporter proteins in nodule tissues. Mol. Plant-Microbe Interact. 20:1040-1047.

Bairoch, A., Boeckmann, B., Ferro, S., and Gasteiger, E. 2004. Swiss-Prot: Juggling between evolution and stability Brief. Bioinform. 5:39-55.

Bevan, M. W. 1984. Binary Agrobacterium tumefaciens vectors for plant transformation. Nucleic Acids Res. 12:8711-8721.

Boutet, E., Lieberherr, D., Tognolli, M., Schneider, M., and Bairoch, A. 2007. UniProtKB/Swiss-Prot: the manually annotated section of the UniProt KnowledgeBase. Methods Mol. Biol. 406:89-112.

Brewin, N. J. 1991. Development of the legume root nodule. Annu. Rev. Cell Biol. 7:191-226.

Bullock, W. C., Fernandez, J. M., and Short, J. M. 1987. XL1Blue: a high efficiency plasmid transforming recA Escherichia coli strain with betagalactosidase selection. BioTechniques 5:376-379.

Carvalho, H. G., Lopes-Cardoso, I. A., Lima, L. M., Melo, P. M., and Cullimore, J. V. 2003. Nodule-specific modulation of glutamine syn- 
thetase in transgenic Medicago truncatula leads to inverse alterations in asparagine synthetase expression. Plant. Physiol. 133:243-252.

Catalano, C. M., Lane, W. S., and Sherrier, D. J. 2004. Biochemical characterization of symbiosome membrane proteins from Medicago truncatula root nodules. Electrophoresis 25:519-531.

Chen, M.-H., Huang, L.-F., Li, H.-M., Chen, Y.-R., and Yu, S.-M. 2004 Signal peptide-dependent targeting of a rice $\alpha$-amylase and cargo proteins to plastids and extracellular compartments of plant cells. Plant Physiol. 135:1367-1377.

Colebatch, G., Desbrosses, G., Ott, T., Krusell, L., Montanari, O., Kloska, S., Kopka, J., and Udvardi, M. K. 2004. Global changes in transcription orchestrate metabolic differentiation during symbiotic nitrogen fixation in Lotus japonicus. Plant J. 39:487-512.

Coque, L., Neogi, P., Pislariu, C., Wilson, K. A., Catalano, C., Avadhani, M., Sherrier, D. J., and Dickstein, R. 2008. Transcription of ENOD8 in Medicago truncatula nodules directs ENOD8 esterase to developing and mature symbiosomes. Mol. Plant-Microbe Interact. 21:404-410.

Crespi, M., and Galvez, S. 2000. Molecular mechanisms in root nodule development. J. Plant Growth Regul. 19:155-166.

Crooks, G. E., Hon, G., Chandonia, J. M., and Brenner, S. E. 2004. WebLogo: a sequence logo generator. Genome Res. 14:1188-1190.

Cullimore, J., and Dénarié, J. 2003. How legumes select their sweet talking symbionts. Science 302:575-578.

Day, D. A., Kaiser, B. N., Thomson, R., Udvardi, M. K., Moreau, S., and Puppo, A. 2001. Nutrient transport across symbiotic membranes from legume nodules. Aust. J. Plant Physiol. 28:667-674.

Doyle, J., and Luckow, M. A. 2003. The rest of the iceberg. Legume diversity and evolution in a phylogenetic context. Plant Physiol. 131:900910

El Yahyaoui, F., Küster, H., Ben Amor, B., Hohnjec, N., Pühler, A., Becker, A., Gouzy, J., Vernié, T., Gough, C., Niebel, A., Godiard, L., and Gamas, P. 2004. Expression profiling in Medicago truncatula identifies more than 750 genes differentially expressed during nodulation, including many potential regulators of the symbiotic program. Plant Physiol. 136:3159-3176.

Emanuelsson, O., Brunak, S., von Heijne, G., and Nielsen, H. 2007. Locating proteins in the cell using TargetP, SignalP, and related tools. Nat. Protocols 2:953-971.

Fedorova, M., van de Mortel, J., Matsumoto, P. A., Cho, J., Town, C. D., VandenBosch, K. A., Gantt, J. S., and Vance, C. P. 2002. Genome-wide identification of nodule-specific transcripts in the model legume Medicago truncatula. Plant Physiol. 130:519-537.

Frühling, M., Albus, U., Hohnjec, N., Geise, G., Pühler, A., and Perlick, A. M. 2000. A small gene family of broad bean codes for late nodulins containing conserved cysteine clusters. Plant Sci. 152:67-77.

Gamas, P., Niebel, F. D. C., Lescure, N., and Cullimore, J. V. 1996. Use of a subtractive hybridization approach to identify new Medicago truncatula genes induced during root nodule development. Mol. Plant-Microbe Interact. 9:233-242.

Ganter, G., Allen, T., and Dunn, K. 1998. The cDNA sequence of nms32/34 (Accession No. AF077405), a gene whose message is specific to the infected cells of alfalfa root nodules (PGR98-155). Plant Physiol. 118:330.

Geurts, R., and Bisseling, T. 2002. Rhizobium Nod factor perception and signalling. Plant Cell 14 (Suppl.):S239-249.

Graham, M. A., Silverstein, K. A., Cannon, S. B., and VandenBosch, K. A. 2004. Computational identification and characterization of novel genes from legumes. Plant Physiol. 135:1179-1197.

Györgyey, J., Vaubert, D., Jimenez-Zurdo, J. I., Charon, C., Troussard, L., Kondorosi, A., and Kondorosi, E. 2000. Analysis of Medicago truncatula nodule expressed sequence tags. Mol. Plant-Microbe Interact. 13:62-71.

Haseloff, J. 1999. GFP variants for multispectral imaging of living cells. Methods Cell Biol. 58:139-151.

Haseloff, J., Siemering, D. R., Prasher, D. C., and Hodge, S. 1997. Removal of a cryptic intron and subcellular localization of green fluorescent protein are required to mark transgenic Arabidopsis plants brightly. Proc. Natl. Acad. Sci. U.S.A. 94:2122-2127.

Hohnjec, N., Perlick, A. M., Pühler, A., and Küster, H. 2003. The Medicago truncatula sucrose synthase gene MtSucS1 is activated both in the infected region of root nodules and in the cortex of roots colonized by arbuscular mycorrhizal fungi. Mol. Plant-Microbe Interact. 16:903-915.

Hohnjec, N., Vieweg, M. F., Pühler, A., Becker, A., and Küster, H. 2005 Overlaps in the transcriptional profiles of Medicago truncatula roots inoculated with two different Glomus fungi provide insights into the genetic program activated during arbuscular mycorrhiza. Plant Physiol. 137:1283-1301.

Journet, E. P., van Tuinen, D., Gouzy, J., Crespeau, H., Carreau, V., Farmer, M. J., Niebel, A., Schiex, T., Jaillon, O., Chatagnier, O., Godiard, L., Micheli, F., Kahn, D., Gianinazzi-Pearson, V., and Gamas,
P. 2002. Exploring root symbiotic programs in the model legume Medicago truncatula using EST analysis. Nucleic Acids Res. 30:5579-5592.

Kaijalainen, S., Schroda, M., and Lindström, K. 2002. Cloning of nodulespecific cDNAs of Galega orientalis. Physiol. Plant. 114:588-593.

Kiss, G. B., Vincze, É., Végh, Z., Tóth, G., and Soós, J. 1990a. Identification and cDNA cloning of a new nodule-specific gene, Nms-25 (nodulin 25) of Medicago sativa. Plant Mol. Biol. 14:467-475.

Kiss, G. B., Csanádi, G., Kálmán, K., Kaló, P., and Ökrész, L. 1993. Construction of a basic genetic map for alfalfa using RFLP, RAPD, isozyme and morphological markers. Mol. Gen. Genet. 238:129-137.

Küster, H., Perlick, A. M., and Pühler, A. 1994. Members of a broad bean nodulin family with partial homologies to the alfalfa nodulin 25 are composed of two types of amino acid repeats flanked by unique amino acid sequence termini. Plant Mol. Biol. 24:143-157.

Küster, H., Quandt, H.-J., Broer, I., Perlick, A. M., and Pühler, A. 1995 The promoter of the Vicia faba L. VfENOD-GRP3 gene encoding a glycine-rich early nodulin mediates a predominant gene expression in the interzone II-III region of transgenic Vicia hirsuta root nodules. Plant Mol. Biol. 29:759-772.

Küster, H., Frühling, M., Pühler, A., and Perlick, A. M. 1996. The modular nodulins Nvf-28/32 of broad bean (Vicia faba L.): alternative exon combinations account for different modular structures. Mol. Gen. Genet. 252:648-657.

Küster, H., Pühler, A., and Perlick, A. M. 1999. Analysis of genes encoding modular nodulins from Vicia hirsuta and Vicia faba. Plant Sci. 149:1-11

Küster, H., Hohnjec, N., Krajinski, F., El Yahyaoui, F., Manthey, K., Gouzy, J., Dondrup, M., Meyer, F., Kalinowski, J., Brechenmacher, L., van Tuinen, D., Gianinazzi-Pearson, V., Pühler, A., Gamas, P., and Becker, A. 2004. Construction and validation of cDNA-based Mt6kRIT macro- and microarrays to explore root endosymbioses in the model legume Medicago truncatula. J. Biotechnol. 108:95-113.

Liu, J., Miller, S. S., Graham, M., Bucciarelli, B., Catalano, C. M., Sherrier, D. J., Samac, D. A., Ivashuta, S., Fedorova, M., Matsumoto, P., Gantt, J. S., and Vance, C. P. 2006. Recruitment of novel calcium-binding proteins for root nodule symbiosis in Medicago truncatula. Plant Physiol. 141:167-177.

Meade, H. M., and Signer, E. R. 1977. Genetic mapping of Rhizobium meliloti. Proc. Natl. Acad. Sci. U.S.A. 74:2076-2078.

Mergaert, P., Nikovics, K., Kelemen, Z., Maunoury, N., Vaubert, D. Kondorosi, A., and Kondorosi, E. 2003. A novel family in Medicago truncatula consisting of more than 300 nodule-specific genes coding for small, secreted polypeptides with conserved cysteine motifs. Plant Physiol. 132:161-73.

Nam, Y. W., Penmetsa, R. V., Endre, G., Uribe, P., Kim, D., and Cook, D. R. 1999. Construction of a bacterial artificial chromosome library of Medicago truncatula and identification of clones containing ethyleneresponse genes. Theor. Appl. Genet. 98:638-646.

Nothwehr, S. F., and Gordon, J. I. 1990. Targeting of proteins into the eukaryotic secretory pathway: signal peptide structure/function relationships. BioEssays 12:479-484.

Oldroyd, G. E., and Downie, J. A. 2006. Nuclear calcium changes at the core of symbiosis signalling. Curr. Opin. Plant Biol. 9:351-357.

Parniske, M. 2000. Intracellular accommodation of microbes by plants: a common developmental program for symbiosis and disease? Curr Opin. Plant Biol. 3:320-328.

Pawlowski, K. 1997. Nodule-specific gene expression. Physiol. Plant. 99:617-631.

Peng, T., and Dickstein, R. 1994. Regulation of plant nodule-specific genes expressed in alfalfa nodules arrested at early-stage of development. Plant Sci. 101:65-73.

Perlick, A. M., and Pühler, A. 1993. A survey of transcripts expressed specifically in root nodules of broadbean (Vicia faba L.). Plant Mol. Biol. 22:957-970.

Pridmore, R. D. 1987. New and versatile cloning vectors with kanamycinresistance marker. Gene 56:309-312.

Quackenbush, J., Cho, J., Lee, D., Liang, F., Holt, I., Karamycheva ,S., Parvici, B., Pertea, G., Sultana, R., and White, J. 2001. The TIGR Gene Indices: analysis of gene transcript sequences in highly sampled eukaryotic species. Nucleic Acids Res. 29:159-164.

Quandt, H. J., Pühler, A., and Broer, I. 1993. Transgenic root nodules of Vicia hirsuta: A fast and efficient system for the study of gene expression in indeterminate-type nodules. Mol. Plant-Microbe Interact. 6:699706.

Quevillon, E., Silventoinen, V., Pillai, S., Harte, N., Mulder, N., Apweiler, R., and Lopez, R. 2005. InterProScan: protein domains identifier. Nucleic Acids Res. 33:W116-120.

Saalbach, G., Erik, P., and Wienkoop, S. 2002. Characterisation by proteomics of peribacteroid space and peribacteroid membrane preparations from pea (Pisum sativum) symbiosomes. Proteomics 2:325-337. 
Sambrook, J., Fritsch, E. F., and Maniatis, T. 1989. Molecular Cloning: A Laboratory Manual, 2nd ed. Cold Spring Harbor Laboratory Press, Cold Spring Harbor, NY, U.S.A.

Schaarschmidt, S., Roitsch, T., and Hause, B. 2006. Arbuscular mycorrhiza induces gene expression of the apoplastic invertase LIN6 in tomato (Lycopersicon esculentum) roots. J. Exp. Bot. 57:4015-4023.

Schultze, M., and Kondorosi, A. 1998. Regulation of symbiotic root nodule development. Annu. Rev. Genet. 32:33-57.

Smit, P., Raedts, J., Portyanko, V., Debelle, F., Gough, C., Bisseling, T. and Geurts, R. 2005. NSP1 of the GRAS protein family is essential for rhizobial Nod factor-induced transcription. Science 308:1789-1791.

Stougaard, J., Joergenson, J. E., Christensen, T., Kuhle, A., and Marcker, K. A. 1990. Interdependence and nodule specificity of cis-acting regulatory elements in the soybean leghemoglobin $l b c 3$ and N23 gene promoters. Mol. Gen. Genet. 220:353-360.

Swiderski, M. R., Zaborowska, Z., and Legocki, A. B. 2000. Identification of new nodulin cDNAs from yellow lupine by differential display. Plan Sci. 151:75-83.

Thoquet, P., Gherardi, M., Journet, E. P., Kereszt, A., Ane, J. M., Prosperi, J. M., and Huguet, T. 2002. The molecular genetic linkage map of the model legume Medicago truncatula: an essential tool for comparative legume genomics and the isolation of agronomically important genes. BMC Plant Biol. 2:1.

Tyerman, S. D., Whitehead, L. F., and Day, D. A. 1995. A channel-like transporter for $\mathrm{NH}_{4}^{+}$on the symbiotic interface of $\mathrm{N}_{2}$-fixing plants. Nature 378:629-632.

Udvardi, M. K., and Day, D. A. 1997. Metabolite transport across symbiotic membranes of legume nodules. Annu. Rev. Plant Physiol. Plant Mol. Biol. 48:493-523.

van Kammen, A. 1984. Suggested nomenclature for plant genes involved in nodulation and symbiosis. Plant Mol. Biol. Rep. 2:43-45.

Vasse, J., de Billy, F., Camut, S., and Truchet, G. 1990. Correlation be- tween ultrastructural differentiation of bacteroids and nitrogen fixation in alfalfa nodules. J. Bacteriol. 172:4295-4306.

Végh, Z., Vincze, É., Kadirov, R., Tóth, G., and Kiss, G. B. 1990. The nucleotide sequence of a nodule-specific gene, Nms-25 of Medicago sativa: its primary evolution via exon-shuffling and retrotransposon-mediated DNA rearrangements. Plant Mol. Biol. 15:295-306.

Vieweg, M. F., Frühling, M., Quandt, H.-J., Heim, U., Bäumlein, H., Pühler, A., Küster, H., and Perlick, A. M. 2004. The promoter of the Vicia faba L. leghemoglobin gene VfLb29 is specifically activated in the infected cells of root nodules and in the arbuscule-containing cells of mycorrhizal roots from different legume and nonlegume plants. Mol. Plant-Microbe Interact. 17:62-69.

von Heijne, G. 1986. A new method for predicting signal sequence cleavage sites. Nucleic Acids Res. 14:4683-4690.

Wienkoop, S., and Saalbach, G. 2003. Proteome analysis. Novel proteins identified at the peribacteroid membrane from Lotus japonicus roo nodules. Plant Physiol. 131:1080-1090.

Zhu, H., Kim, D.-J., Baek, J.-M., Choi, H.-K., Ellis, L. C., Küster, H., McCombie, W. R., Peng, H.-M., and Cook, D. R. 2003. Syntenic relationships between Medicago truncatula and Arabidopsis reveal extensive divergence of genome organization. Plant Physiol. 131:10181026

\section{AUTHOR-RECOMMENDED INTERNET RESOURCES}

DFCI Medicago truncatula Gene Index: compbio.dfci.harvard.edu/tgi Medicago genome sequencing project: www.medicago.org/genome

National Center for Biotechnology Information website: www.ncbi.nlm.nih.gov

University of California-Berkeley WebLogo3 software: weblogo.berkeley.edu/logo.cgi 